\title{
Relations between asbestos exposure and lung cancer SMRs in occupational cohort studies
}

\author{
F D K LIDDELL AND J A HANLEY
}

From the Department of Epidemiology and Biostatistics, McGill University, Montreal, Canada

ABSTRACT It has long been accepted that excessive exposure to asbestos may produce lung cancer but not that there is a consistent "biological gradient." This can only be evaluated reliably in studies where, for every individual, exposure has been measured in terms of both duration and intensity. Even now, there are only at most eight such cohort studies of asbestos workers, while formal methods of analysis have been available only recently. These methods, applied in these studies, yield good evidence that the "exposure-response" relation between accumulated exposure to asbestos and standardised mortality ratios (SMRs) for lung cancer may be taken as linear, but that at zero exposure the lung cancer SMR is not always unity-not surprising, because of well known difficulties with the choice of reference population and selection problems. This leads to a concept of "relative slopes" that take account of the background mortality in the cohort and make what appears to be the best use of the available data. Other approaches to the same data, and indeed to all cohort data known, are also considered. Each study is examined as closely as is possible in a short review, and the concepts of linearity and relative slopes appear justified. The relative slopes $(b / a)$ in the line $\mathrm{SMR}=a[1+(b / a) \cdot($ exposure $)]$ vary much more widely than can be accounted for by differences in epidemiological methodology; as discussed elsewhere, reasons for the variation seem to lie rather in type and dimensions of asbestos fibre, industrial process, etc. Slopes in the line $\mathrm{SMR}=1+b_{1} \cdot$ (exposure) vary about twice as much as do the relative slopes.

In the past few years several reviews of the health effects of asbestos have been published, most having been commissioned by government agencies. ${ }^{1-6}$ In all these reviews much effort was concentrated on the evaluation from cohort studies of "exposureresponse" relations for lung cancer. Two approaches to such evaluation are possible. The first ${ }^{7}$ needs only the following data: the overall standardised mortality ratio (SMR) ${ }^{8}$ for lung cancer and a single assessment of the average exposure to asbestos of the complete cohort. This attractive simplicity is, however, counterbalanced by the need to rely on two assumptions: (1) that the group studied would have had the same mortality experience as the reference population had there been no occupational exposure to asbestos; and (2) that the exposure-response relation is linear. As we shall show, grave doubts often surround assumption (1), although assumption (2) is probably reasonable.

Received 13 August 1984

Accepted 17 September 1984
The second approach requires estimates of exposure for each individual subject, preferably in terms of both duration and concentration. It is then necessary to classify the cohort, in terms of levels of exposure to asbestos, into a number of subcohorts, and to obtain for each the average level of exposure and the SMR. Thereafter reliable evaluation requires certain formal methods of curvefitting, apparently first used in $1982 .^{9}$ In the present paper we exploit these methods ${ }^{9 a}$ in those cohort studies of asbestos workers where adequate data have been provided.

Although there have been 30 or more reports of cohort mortality studies of asbestos workers, even now there are only seven studies in which exposure has been calculated for every subject in terms of both duration and concentration. In these seven the authors have classified their cohort, in terms of levels of exposure to asbestos (accumulated over some specified period), into a number (which we call h) of subcohorts. For the $i$-th subcohort, the data become: an estimate of the mean exposure $\left(x_{i}\right)$, the number of observed deaths $\left(D_{i}\right)$, and the number 
expected $\left(E_{i}\right)$ on the basis of a "person-years" analysis making use of the mortality in some population, selected as reference, and the derived $\operatorname{SMR}_{\mathrm{i}}(=$ $D_{i} / E_{i}$, taken as 1 , not 100 , when $D=E$ ). A plot of the subcohort lung cancer SMRs against $x_{i}$ (most have been published) usually shows the relation to be well described by a straight line, despite variation in methodology that might have been expected to distort the basic pattern, and despite the fact that there is no immediately obvious biological explanation. It has been pointed out, however-see, for example, Liddell' - that the line does not necessarily pass through (or even close to) an SMR of unity for zero exposure. This is in no way surprising because of difficulties over the choice of a truly relevant reference population and in selection and other related factors.

In this paper we fit formally ${ }^{9 a}$ the two parameter line $(\mathrm{SMR}=a+b \cdot \mathrm{x})$ and the one parameter line $(\mathrm{SMR}=1+b \cdot \mathbf{x})$ to the data for all appropriate cohort studies. We give the goodness of fit statistics for the fitted lines, and use them to yield a formal test of whether $\hat{a}$ (the value of the parameter $a$ fitted by maximum likelihood (ML)) may be taken as unity. We exploit and justify the concept of "relative slopes," which is of particular importance when $\hat{a}$ is far from unity, and show that the relative slopes may be steeper or shallower than the slopes fitted in the one parameter model.

\section{Materials and methods}

Table 1 lists the seven investigations of cohorts of asbestos workers in which exposure has been estimated, for each member of the cohort, both by dura- tion and by concentration. (A recent report ${ }^{19}$ on an asbestos-cement factory in Ontario is not included; see discussion.) The stimulus variable, $x$, was always accumulated exposure to asbestos (measured in millions of particles per cubic foot, multiplied by years, or mpcf.y-but accumulated over different periods according to the study design). Cohorts A1/P and A1/M were subgroups of cohort A1, and study A2 was of the same cohort updated but restricted by country of residence. The codes $\mathrm{C} / 1$ and $\mathrm{C} / 2$ refer to different forms of a priori analysis ${ }^{8}$ of the same cohort. Studies E(a) and E(b) were carried out independently, $\mathrm{E}(\mathrm{a})$ - much the larger-being the more recent. Added to table 1 is an inquiry (G) based only on duration of employment. Code $G / 1$ follows Nicholson ${ }^{4}$ in basing observed deaths on "best evidence" of cause and in omitting from the fit, on grounds of "saturation" effects (see discussion), those who had been employed two years or more; code G/2 uses the causes coded on death certificates and includes all men in the cohort.

When fitting a straight line of the form SMR = $\mathrm{D} / \mathrm{E}=\boldsymbol{a}+\boldsymbol{b} \cdot \mathrm{x}$, multiplying through by $\mathrm{E}$, to get $\mathrm{D}=$ $a \cdot E+b \cdot(x E)$, is a help, if only in showing the kinship with regression analysis. The usual assumptions are that $\mathrm{D}$ has Poisson variation, and $\mathrm{E}$ may be taken as based on such large numbers as to be without error (see also Berry ${ }^{20}$ ). As $\mathrm{x}$ is the mean exposure for a subcohort, it has a fairly small sampling error; thus (xE) may also be taken as virtually error free. We have shown elsewhere ${ }^{\text {a }}$ how the ML fit may be obtained by an iterative process of weighted least squares (IWLS) regression. The ML fit of the two parameter model-that is, without constraint on $a$-leads to estimates of $a$ and $b$ that are termed $\hat{a}$

Table 1 The cohorts evaluated

\begin{tabular}{|c|c|c|}
\hline Study code & Brief description and reference & $E L C^{*}$ \\
\hline $\begin{array}{l}\text { A1 } \\
\text { A1/P } \\
\text { A1/M } \\
\text { A2 }\end{array}$ & $\begin{array}{l}1464 \text { male retirees from a US asbestos company }{ }^{+10} \\
1026 \text { former production workers in } A 1^{10} \\
438 \text { former maintenance/service workers in } A 1^{10} \\
1075 \text { male retirees, as in A1 but up-dated, with some forced exclusions }\end{array}$ & $\begin{array}{r}24 \\
17 \\
7 \\
23\end{array}$ \\
\hline B & 5645 male workers in asbestos-cement plants New Orleans, LA $^{12}$ & 49 \\
\hline $\begin{array}{l}\mathrm{C} / 1 \\
\mathrm{C} / 2\end{array}$ & $\begin{array}{l}10939 \text { male miners and millers in Quebec, analysed in terms of exposure accumulated to age } 45^{13} \\
3105 \text { men in } \mathrm{C} 1 \text {, but with at least } 20 \text { years' gross service }\end{array}$ & $\begin{array}{r}197 \\
65\end{array}$ \\
\hline $\mathrm{D}$ & 4137 male workers at an asbestos plant in PA, in mainly textile manufacture ${ }^{14}$ & 50 \\
\hline $\begin{array}{l}E(a) \\
E(b)\end{array}$ & $\begin{array}{l}2543 \text { male workers (white and black) at an asbestos textile plant in Charleston, } \mathrm{SC}^{15} \\
768 \text { white male workers in the same plant as } \mathrm{E}(\mathrm{a})^{16}\end{array}$ & $\begin{array}{r}29 \\
7\end{array}$ \\
\hline $\mathbf{F}$ & 3641 male workers in a friction products and packings plant in $\mathrm{CT}^{17}$ & 49 \\
\hline $\begin{array}{l}\mathrm{G} / 1 \\
\mathrm{G} / 2\end{array}$ & $\begin{array}{l}632 \text { men who had worked less than two years in an amosite insulation products facility in Paterson, } \mathrm{NJ} \text {, with } \\
\text { cause of death based on "best evidence" } 18 \\
820 \text { men, as in G/1 together with } 188 \text { employed two years or more, with cause of death based on death } \\
\text { certificate } 18\end{array}$ & $\begin{array}{l}16 \\
22\end{array}$ \\
\hline
\end{tabular}

* Expected numbers of deaths from lung cancer-an indicator of reliability.

†P E Enterline et al, paper presented at American Academy of Occupational Medicine at Pittsburg, 1972. 
and $\hat{b}$. In the one parameter model, where $a$ is constrained to unity, the ML estimate of the single (slope) parameter is called $\hat{b}_{1}$.

It has been thought that ML would also minimise the goodness of fit statistic for deviations from linearity - that is, deviations of $D$ from the quantity $\hat{a} \cdot E+b \cdot(x E)$ [or from $E+\hat{b}_{1} \cdot(x E)$ ] calculated from the fitted line-but this is true only asymptotically (Hanley, Liddell). Although the "minimum $x^{2 "}$ approach is in some ways more appealing, it is computationally more difficult than IWLS, and less flexible. Further, it was found, from the examination of results in all 13 sets of data listed in table 1, that the two approaches led to sufficiently similar estimates of all statistics that the use of one rather than the other would be most unlikely to mislead. The IWLS, or ML, approach is used in all of what follows; versions of tables 2 and 3 based on minimum $\chi^{2}$ are, however, available on request.

\section{Results}

\section{SMRS AT LOW EXPOSURES}

In consideration of whether the lung cancer SMR = 1 when $x=0$ a first step is to examine SMRs for the subcohorts with least severe exposure. These are given in table 2, together with p-values for an exact (two sided) test of deviation from unity. ${ }^{21}$ The SMRs for the least severely exposed were raised for studies $A$ (all phases, particularly $A / 2), E(a), E(b), F, G / 1$, and $G / 2$; they were depressed for studies $B$ and $D$; and they were close to unity only for study $C$. Thus it would appear likely that several of the lines might not yield SMR close to 1 when $x=0$.

A test of whether the intercept, $a$, fitted in the two parameter model, differs from unity may be based on the difference between the goodness of fit statistic from the one parameter model (with $\mathrm{df}=\mathbf{h}-1$ ) and the corresponding statistic for the two parameter model $(\mathrm{df}=\mathrm{h}-2)$; this difference may be taken as a $\chi^{2}$ statistic with $1 \mathrm{df} .^{9 a}$ Values of this statistic, and of $\hat{a}$. have been added to table 2 . The values of this $\chi^{2}$ were high enough for studies $D, F$, and $G$ (both versions) that the hypothesis $a=1$ could be rejected with considerable confidence. They were suggestive for studies $B$ and $A / 2$, but, because there are usually few deaths, "significant" test statistics are uncommon, and some judgment is necessary in interpretation.

\section{RELATIVE AND ONE PARAMETER SLOPES}

Rearrangement of terms gives SMR $=a[1+(b /$ a) $\cdot \mathrm{x}$ ], which (with some reservations about the use of ratios of SMRs for relative risks, RRs) ${ }^{9 a}$ leads to $R R$ $=1+(b / a) \cdot x$. The term $(\hat{b} / \hat{a})$ was called the "relative slope" by Liddell, ${ }^{9}$ Acheson and Gardner, ${ }^{6}$ and others. The ML estimates of the one parameter slope $\hat{b}_{1}$, and of the relative slope $(\hat{b} / \hat{a})$, for one analysis (either the latest or the most reliable) from each investigation have been determined (table 3 ). For studies D, F, and G/2 (where $a$ was clearly not unity), $\hat{b}_{1}$ and $\hat{b} / \hat{a}$ differed markedly, being in the ratios $0 \cdot 3: 1$ and $4 \cdot 0: 1$ for $D$ and $G / 2$, and being of opposite sign for $F$. In studies $A / 2$ and $B$ (where the

Table 2 SMRs for subcohorts with least severe exposure, and intercepts â of two parameter fits, with test statistics

\begin{tabular}{|c|c|c|c|c|c|c|}
\hline Study & $\begin{array}{l}\text { Maximum exposure } \\
\text { for subcohort }\end{array}$ & Average exposure & $S M R$ & p-value & Test statistic $\dagger$ & $\hat{a}$ \\
\hline $\begin{array}{l}\text { A1 } \\
\text { A1/P } \\
\text { A1/M } \\
\text { A2 }\end{array}$ & $\begin{array}{l}125 \text { mpcf.y } \\
125 \text { mpcf.y } \\
125 \text { mpcf.y } \\
125 \text { mpcf.y }\end{array}$ & $\begin{array}{l}55.7 \text { mpcf.y } \\
55.7 \text { mpcf.y } \\
55.7 \text { mpcf.y } \\
62 \text { mpcf.y }\end{array}$ & $\begin{array}{l}1.68 \\
1.47 \\
2.19 \\
1.98\end{array}$ & $\begin{array}{l}0.0513 \\
0.2775 \\
0.0892 \\
0.0095\end{array}$ & $\begin{array}{l}1.0582 \\
0.8125 \\
0.1757 \\
1.5080\end{array}$ & $\begin{array}{l}1.40 \\
1.46 \\
1.39 \\
1.46\end{array}$ \\
\hline B & 10 mpcf.y & 5 mpcf.y & 0.77 & 0.2930 & $2 \cdot 2931$ & 0.69 \\
\hline $\begin{array}{l}\mathrm{C} / 1 \\
\mathrm{C} / 2\end{array}$ & $\begin{array}{l}1 \text { mpcf.y } \\
100 \text { mpcf.y }\end{array}$ & $\begin{array}{l}0.5 \text { mpcf.y } \\
67 \text { mpcf.y }\end{array}$ & $\begin{array}{l}0.96 \\
1 \cdot 21\end{array}$ & $\begin{array}{l}0.9335 \\
0.3564\end{array}$ & $\begin{array}{l}0.2015 \\
0.0108\end{array}$ & $\begin{array}{l}0.96 \\
1.01\end{array}$ \\
\hline D & 10 mpcf.y & 5 mpcf.y & 0.67 & 0.0654 & $6 \cdot 3918$ & 0.53 \\
\hline $\begin{array}{l}E(a) \\
E(b)\end{array}$ & $\begin{array}{l}10 \text { mpcf.y } \\
9.13 \text { mpcf.y }\end{array}$ & $\begin{array}{l}5 \text { mpcf.y } \\
4.56 \text { mpcf.y }\end{array}$ & $\begin{array}{l}1.43 \\
2.23\end{array}$ & $\begin{array}{l}0.0688 \\
0.0607\end{array}$ & $\begin{array}{l}0.0905 \\
0.7629\end{array}$ & $\begin{array}{l}1.09 \\
1.62\end{array}$ \\
\hline $\mathbf{F}$ & 10 mpcf.y & 5 mpcf.y & 1.67 & 0.0005 & $12 \cdot 8442$ & 1.63 \\
\hline G/1 & $\begin{array}{l}1 \text { month } \\
2 \text { months } \\
3 \text { months } \\
1 \text { month } \\
2 \text { months } \\
3 \text { months }\end{array}$ & $\begin{array}{l}* \\
\overline{-} \\
= \\
-\end{array}$ & $\begin{array}{l}2.94 \\
2 \cdot 88 \\
2 \cdot 84 \\
2 \cdot 31 \\
2 \cdot 40 \\
2 \cdot 40\end{array}$ & $\begin{array}{l}0.0984 \\
0.0063 \\
0.0003 \\
0.2858 \\
0.0415 \\
0.0058\end{array}$ & $12 \cdot 9974$ & $2 \cdot 28$ \\
\hline
\end{tabular}

* Uniform concentrations of $35(\mathrm{f} / \mathrm{ml})$ assumed for all workers ${ }^{4}$ suggest an average exposure of $1.5(\mathrm{f} / \mathrm{ml}) . \mathrm{y}$, or approximately $0.5 \mathrm{mpcf} . \mathrm{y}$, for those employed less than one month.

26 Difference between goodness of fit statistics with $(h-1)$ and $(h-2)$ df, which may be used as a $\chi^{2}$ statistic, with 1 df, to test the hypothesis $\hat{a}=1$. 
$\chi^{2}$ test of $a=1$ was suggestive), the ratios were $1 \cdot 9: 1$ and $0 \cdot 5: 1$; the ratios were $1 \cdot 9: 1$ in study $E(b)$ (where the SMR for the subcohort with lowest exposure-only 5 mpcf.y on average-was $2 \cdot 23$, but based on very small numbers), and $0 \cdot 9: 1$ and $1 \cdot 1: 1$ for studies $\mathrm{C} / 1$ and $\mathrm{E}(\mathrm{a})$ (where there was no strong reason for not accepting $a=1$ ).

\section{GOODNESS OF FIT}

We examined the goodness of fit of both the two parameter and the one parameter lines for the eight cohorts forming the subject of table 3 . The sum of the goodness of fit statistics for the deviations from the lines fitted with two parameters is 17.94 with $30 \mathrm{df}$, giving no indication of inadequate fit; the one doubtful case is study $B(7 \cdot 91$ with $3 \mathrm{df})$. For the one parameter fits, the corresponding statistic was 55.01 with $38 \mathrm{df}$, not particularly surprising in total, but including two rather high values of the statistic for studies $F$ and $G / 2(15 \cdot 10$ with $4 \mathrm{df}$; and $16 \cdot 27$ with $6 \mathrm{df})$, as well as the borderline study B (10.20 with $4 \mathrm{df})$.

\section{ESTIMATES OF RELATIVE SLOPE}

Table 3 summarises the point estimates, with lower and upper $90 \%$ confidence limits, of relative slope (b/a) for the eight cohorts already identified; the estimates calculated from the original values of $\mathbf{x}$ (in mpcf.y) have been multiplied by 100 for ease of presentation. The estimates are not strictly comparable, particularly because of the different periods (1) of follow up and (2) over which exposures were accumulated-see references ${ }^{14}$ and ${ }^{15}$ for the effects of different choices of periods in the same cohort.

\section{Discussion}

It was stated above that the Peto method ${ }^{7}$ relied on two assumptions. These are examined, together with

Table 3 Estimates of one parameter and relative slopes*

\begin{tabular}{|c|c|c|c|c|}
\hline \multirow[t]{3}{*}{ Study } & \multirow{3}{*}{$\begin{array}{l}\begin{array}{l}\text { One-parameter } \\
\text { model }\left(b_{1}\right)\end{array} \\
\begin{array}{l}\text { (Point } \\
\text { estimate })\end{array}\end{array}$} & \multicolumn{3}{|c|}{ Relative slope (b/a) } \\
\hline & & \multirow{2}{*}{$\begin{array}{l}\text { (Point } \\
\text { estimate) }\end{array}$} & \multicolumn{2}{|c|}{ ( $90 \%$ confidence interval) } \\
\hline & & & Lower & Upper \\
\hline $\begin{array}{l}\text { A2 } \\
B \\
C 11 \\
D \\
E(a) \\
E(b) \\
F \\
G / 2 \dagger\end{array}$ & $\begin{array}{r}0.654 \\
0.329 \\
0.150 \\
1.653 \\
6.724 \\
13.224 \\
0.504 \\
13.503\end{array}$ & $\begin{array}{r}0.353 \\
0.658 \\
0.164 \\
5 \cdot 101 \\
5.863 \\
6.896 \\
-0.489 \\
3.343\end{array}$ & $\begin{array}{l}0.088 \\
0.204 \\
0.090 \\
1.756 \\
2.071 \\
1.342 \\
- \text { ve } \\
0.967\end{array}$ & $\begin{array}{r}1 \cdot 170 \\
1.559 \\
0 \cdot 264 \\
15 \cdot 078 \\
16 \cdot 549 \\
57 \cdot 592 \\
0 \cdot 414 \\
8 \cdot 183\end{array}$ \\
\hline
\end{tabular}

${ }^{*}$ Rate of increase $(\times 100)$ of excess lung cancer risk per unit mpcf.y.

tAssuming the conversion factor generally adopted by Nicholson ${ }^{4}$ that is, $3(\mathrm{f} / \mathrm{ml}) / \mathrm{mpcf}-$ and so taking the uniform concentration as $35 / 3$ mpcf for all workers; see footnote to table 2 . the plausibility of taking account of "background mortality" by means of relative slopes. The concept of "saturation" is also reviewed.

The terms SMR and relative risk (RR) have been used interchangeably. ${ }^{45}$ It is obvious from our findings that this usage is incorrect. Indeed, it has been well known for many years that in any occupational cohort study the choice of reference population is vital. The need to make internal comparisons, by means of relative slopes, put forward no later than 1982," has been recognised by Acheson and Gardner in their latest review for the Health and Safety Executive, ${ }^{6}$ by McDonald, ${ }^{22}$ by Sir Richard Doll (personal communication), and by others..$^{14} 1517$ Acheson and Gardner, ${ }^{6}$ paraphrasing our earlier arguments," point out that as, for each study, SMRs were calculated based on an external standard, the constraint that forces the line through an SMR of 1 at zero exposure is tantamount to assuming that the chosen reference population and the asbestos workers had a similar experience-other than exposure to asbestos-in respect of all factors (including smoking) with a bearing on the incidence of lung cancer. They believe that it is unlikely that this will have been true in any particular study, and they prefer the relative risk approach-and made use of our estimates $^{9}$ of relative slopes- - "because it does not involve any assumption about the appropriateness of the reference population, and also describes the empirical data more closely," 6 but it does mean assuming that the background mortality, $a$, was the same in every subcohort (G Berry, personal communication).

In our section on goodness of fit, the $\chi^{2}$ accumulated for all two parameter fits $(17.94$, with $30 \mathrm{df})$ gives no indication of inadequate fit; one fairly low p-value may be acceptable when all the others are so high. There seems little doubt that linearity can usually be accepted, but only in the two parameter model-that is, when not assuming $a \equiv 1$. There are other possibilities-linearity has not been proved, but the comments that follow on each of the specific cohorts and their analysis suggest that linearity, with $(\hat{b} / \hat{a})$ as an estimate of relative slope, is not an unreasonable explanation of the general phenomenon.

Nicholson used the findings from study $G$ to postulate the appearance of a saturation effect for the risk of deaths from lung cancer after two years of employment. ${ }^{4} \mathrm{He}$ stated that "this could result from selective early removal of individuals at particular risk as, for example, heavy cigarette smokers, or by the competing risk of death from asbestosis in the higher exposure categories." There are several arguments against this effect. Firstly, there is no evidence of selective early removal of those at particu- 
lar risk. Secondly, the deaths from asbestosis even in the group with longest employment were still only about $15 \%$ of all deaths. Thirdly, the estimated average exposure of those who had worked for more than two years was only about 50 mpcf.y, whereas in many other studies there was no evidence of a corresponding saturation at much higher levels of accumulated exposure. Fourthly, the two parameter goodnes of fit statistic for the data from study G/2 was 3.29 with $5 \mathrm{df}$, bringing fairly strong evidence that a straight line was a satisfactory fit. Further findings that Nicholson considered evidence of saturation came from one study-see study $M$ below-where in fact there was no dose-response relation, so that he postulated that even those working in the cleanest departments, such as research, non-asbestos products or wet processes, had been saturated. ${ }^{4}$

The estimates of slope made by Nicholson ${ }^{4}$ were always steeper than those of Liddell'; according to Acheson and Gardner the reason probably lay in that "the populations used for comparison had a more favourable experience than the workforce under consideration in respect of other carcinogens with a bearing on the incidence of lung cancer, eg, smoking habits." Another reason for the consistent difference lay in the adjustments Nicholson made for saturation which, as explained above, do not seem acceptable. It is important, however, to examine each finding separately in order to balance the evidence. The following notes deal with each study in turn; a number prefixed AG indicates the study number given by Acheson and Gardner ${ }^{6}$ on their pages $10-11$. The estimates by Nicholson ${ }^{4}$ are also given where available; these have been remultiplied by $3(\mathrm{f} / \mathrm{ml}) / \mathrm{mpcf}$ - the factor usually used by Nicholson ${ }^{4}$ - to convert exposures back to the (usually) original mpcf.y terms, so that they are in the same dimensions as ours.

Study $A$ (including AG3 and AG5) - In their earliest report on Study A1, the authors ( P E Enterline et al, Pittsburgh) noted that, for all retirees, the lung cancer SMR was 1.68 in the subcohort with the least severe exposure (cf table 2 ). This obviously caused them concern, and they also presented findings from a rather small but "completely parallel study" in which there had been no exposure to asbestos or other suspected carcinogenic dust; here the lung cancer SMR was $9 / 5.3=1.70$, almost identical. Values of $\hat{a}$ for studies $\mathrm{A} 1, \mathrm{~A} 1 / \mathrm{P}, \mathrm{A} 1 / \mathrm{M}$, and $\mathrm{A} 2$ were fairly consistent (see table 2), but the values of $\chi^{2}$ with $1 \mathrm{df}$ for the improvement in fit of the two parameter model over the one parameter model were not impressive. For the four facets of this study, however, the ratios of the relative slope to the one parameter slopes were: $0.57,0.34,0.65$, and
0.54. Nevertheless, five years ago, Acheson and Gardner accepted that for studies A1/P and A1/M they could take $a$ as unity, ${ }^{12}$ and their "fitted" one parameter slopes were close to $3 / 800$ and $10 / 800$ (as estimated from their fig $11^{\prime}$ ). The first of these estimates is nearly $1 \frac{1 / 2}{2}$ times steeper than that obtained by ML. For A2, the authors' own (unweighted) fit of SMR to $x$ yielded a value of $\chi^{2}$ almost identical to that for the one parameter ML model but with only $3 \mathrm{df}$, thus gaining nothing despite using up an extra degree of freedom." The slope estimate $(0.90)$ by Nicholson $^{4}$ was also adjusted (arbitrarily) because the subjects had been retirees.

Study $B$ (AG10) shows the reverse phenomenon-that is, $\hat{a}$ less than 1 ; this is by no means unusual in the state of Louisiana (P E Enterline, $\mathrm{H}$ Weill, personal communications). Interestingly, the number of lung cancer deaths, 51 , in the complete cohort was only slightly greater than the total expected, 49.2. Again the relative slope is changed from the one parameter slope, being just twice as steep. The values of $\chi^{2}$ with 3 and $4 \mathrm{df}$ for deviations from linearity are associated with $p$ values of 0.048 and 0.037 . The evidence appears slightly in favour of the two parameter model. The deviations from linearity are quite irregular and give no indication of any simple model, but some lack of linearity cannot be ruled out on the evidence presented. Nicholson ${ }^{4}$ ignored this study.

Study $C$ (AG1)-The definition of the cohort meant that by the end of the study period most members were not still employed in chrysotile production. It was, therefore, not unexpected that the values of $\hat{a}$ were close to unity for $\mathrm{C} / 1$ and $\mathrm{C} / 2$, so that the relative and one parameter slopes were similar both for $\mathrm{C} / 1$ and for $\mathrm{C} / 2$. Nicholson's estimate of slope for $\mathrm{C} / 1$ was close-that is, $0 \cdot 18 .^{4}$ Nevertheless, as the case-referent-within-a-cohort analysis is considered the most appropriate for determining RRs, we prefer the point estimate 0.136 from Berry, ${ }^{23}$ with $90 \%$ confidence limits 0.058 and 0.237 , from Liddell. ${ }^{9}$ An important finding was that in $\mathrm{C} / 2$, the point estimate of $(b / a)$ was 0.203 (with $90 \%$ confidence interval 0.064 to 0.493 ). This shows the importance of asbestos concentration in the evaluation of exposure, because in this portion of the study the workers in the four subcohorts, having been selected as with at least 20 years' gross exposure, had had similar average durations of exposure.

Study $D$ (AG11)-The subcohort with exposure average 5 mpcf.y had SMRs well below unity not only for lung cancer but also for other respiratory disease including pneumoconiosis $(0.61)$, cerebrovascular diseases $(0.78)$, and "other known causes" $(0 \cdot 73)$. McDonald et al thought it likely that 
the explanation lay in lack of comparability with the population of the relevant state, Pennsylvania. ${ }^{14}$ This was confirmed by the low value of $\hat{a}$; naturally, there was a big difference in the slopes, the relative slope being three times as steep as that from the one parameter model. This and the following study were published well after the review by Nicholson. ${ }^{4}$

In Study E(a) (AG8) the lowest exposure subcohort had high SMRs for most groups of causes, and an explanation corresponding to that for study $D$ was suggested in relation to South Carolina. Nevertheless, because here the value of $\hat{a}$ was only 1.09 , the relative slope was nearly as steep as the one parameter slope. For comparing this and study $\mathrm{D}$, the methodology was identical. The similarity of the relative slopes, based on the two parameter model, is rather more in keeping with epidemiological expectation than the wide divergence of the one parameter slopes. For comparison with study $E(b)$, reanalysis of data obtained by the same methods as for that study gave the point estimate $(\hat{b} / \hat{a})=5 \cdot 107$.

Study $E(b)$ (AG7)-Here, Dement et al spent considerable effort on the choice of reference population (USA white men), rejecting several alternatives. ${ }^{16}$ The choice as reference of white men in the county in which the factory was sited would have lowered the SMR for the least exposed group from 2.23 to around 1.3 ; however, this choice was rejected on grounds that do not find universal favour, ${ }^{6}$ particularly as, on the basis selected, $\hat{a}=$ 1.62 ; although the $1 \mathrm{df} \chi^{2}$ was only $0 \cdot 76$, numbers were small (only 26 lung cancers in all). The relative slope was only half as steep as the one parameter slope, but with a wide confidence interval due to the smallness of the study and because $h=4$ only. Dement et al had assumed 365 working days in a year, ${ }^{16}$ but Liddell had not appreciated this and his estimate (equivalent to $4 \cdot 8$ ) was incorrect ${ }^{9}$-it should have been as in our table 3 . The estimate (equivalent to 15.8 ) by Nicholson ${ }^{4}$ was based on $a=$ 1 , and apparently on a saturation argument.

Study $F$ was unusual in that such a high proportion of the workers were in the group with least severe exposure (average 5 mpcf.y) so that their 55 deaths from lung cancer (out of 73 in all) led to an SMR of 1.67 that dominated the analysis. As pointed out by McDonald et al selection was surely an important factor here. ${ }^{17}$ The more severely exposed groups accounted for only 18 deaths from lung cancer $($ SMR $=1 \cdot 12)$, so that-at face value-a two parameter fit gave a negative slope. The $90 \%$ confidence limits on the estimate of $(b / a)$ embrace zero, and there is little evidence for increased risk of lung cancer with more severe exposure to asbestos in this plant. Nicholson's review ${ }^{4}$ was published before the report of this study.
Study G(AG14)-In his criteria document for the Swedish government, Nicholson placed much weight on the material of study $G / 1$, although not only were the numbers of deaths determined from the best evidence ("BE") of cause of death (and therefore manifestly not comparable with the expectations that are based on causes coded on death certificates) but also those who had been employed for less than one month had a lung cancer "SMR" of 2.94. ${ }^{4}$ Even Seidman et al $^{18}$ placed considerable emphasis on the high SMRs for exposure of extremely short duration, and based further argument on the groups with duration of more than three, but fewer than 24, months; table 2 shows that all three groups with the shortest exposures had SMRs for lung cancer that really could not be accounted for on any of the hypotheses nowadays under serious consideration, whether lung cancer deaths were based on $\mathrm{BE}$ or on death certificate coding. In study $G / 2$ the one parameter slope was four times as steep as the relative slope. Nicholson's estimate (27.3, twice as steep again) was based on $a=1$ (which has been seen to be palpably false), on best evidence of cause of death (which led to considerable inflation), and ignoring those employed for more than two years, on grounds of saturation. ${ }^{4}$ As already pointed out, however, the goodness of fit statistic suggests a perfectly acceptable linear fit-with two parameters. The (invalid) fit of $\mathrm{D}$ (on $\mathrm{BE})$ to $(a+b \cdot \mathrm{x}) \mathrm{E}$ for the complete cohort-that is, study $\mathrm{G} / 1$ extendedwould have led to a similar conclusion.

Six other studies, none of which met the criteria for inclusion in this paper, are discussed briefly below. Several of Nicholson's estimates are considered too high because he not only took $a=1$ but also based some of the slopes on groups with low exposure to avoid saturation effects; neither assumption appeared justified wherever data were available for their evaluation.

Study $H$ (AG2) was of Quebec miners and millers but was a much smaller investigation than study $C$, having been based on workers with long exposure at Thetford Mines only; individual exposures were not assessed. ${ }^{24}$ Nicholson's 1981 estimate (0.45) does not appear to be justified by his material ${ }^{4}-$ a major reason being an allowance for saturation-and falls well outside the limits cited for study C; Liddell's independent estimate ${ }^{y}$ in 1982 from the same data was $0 \cdot 135$, close to that for study $C$ itself, probably because assumption (1) was not grossly violated.

Study J (AG15) was a large study of insulation workers in North America, ${ }^{25}$ but it suffered from there being only global, retrospective éstimates of exposure. In 1981 Nicholson made an estimate of slope $5 \cdot 1,{ }^{4}$ and whereas we believe this to be too steep we are unable to offer any reliable estimate of 
our own, although in 1982 Liddell offered a guess of $4 \cdot 5,{ }^{9}$ based on Nicholson's estimates of exposures. ${ }^{4}$

Study $K$ (AG13) was of factory workers in London, ${ }^{26}$ but information on exposure is coarse on duration and unreliable as to intensity. The large difference between the sexes is unexplained, but many of the few female factory workers were probably much heavier smokers than the general population of women. Assumption (1) did not seem to apply, particularly for women, and the 1981 estimates of slope by Nicholson of 3.9 for men and $25 \cdot 2$ for women, ${ }^{4}$ were considered too high by Liddell, ${ }^{9}$ who suggested $1 \cdot 2-3 \cdot 3$ and $8 \cdot 1$, respectively.

Study L (AG6) was of asbestos textile workers in a factory in Lancashire. ${ }^{27}$ Schneiderman et al found difficulty in reconciling the differences between the earlier and later subcohorts in the same facility, ${ }^{5}$ for which Nicholson had quoted slopes of 0.21 and 2.4 respectively. ${ }^{4}$ We agree about the difficulties of reconciliation but cannot find adequate detail for making alternative estimates.

Study $M$ (AG4) has apparently not been published; although discussed by Nicholson, ${ }^{4}$ insufficient detail is quoted and the methodology is unclear. Nevertheless, the four groups of workersranked from least to most heavily exposed (in the ratios 1:2:4:8)-had SMRs of 3.06, 2.46, 2.63, and 3.45 , respectively, based on a total of only 35 deaths. This renders any analysis inappropriate. Nicholson, using only the first of these SMRs and assuming $a=1$, obtained a slope of $3 \cdot 3 .^{4}$ The relative slope is clearly shallow, although a slope based on the Peto method' might be around 1.5.9

Study $N$ (AG9) was of workers manufacturing friction materials using asbestos. Berry and Newhouse found no association of excess risk with duration of employment, ${ }^{28}$ and although giving an estimate of slope from a case-referent-within-a-cohort analysis, they stated that it "could have arisen by chance"; it seems appropriate to take it as effectively zero.

To be complete, we mention two recent studies of asbestos-cement workers ${ }^{1929}$; the former updates AG12. They were small (ELCs of perhaps 10 and 20 , respectively) with high lung cancer rates (SMRs for complete cohorts around 5 and 2), not systemically related to asbestos exposure. Both workforces suffered inordinately from mesothelioma (probably because the factories used amphiboles as well as chrysotile), and the most heavily exposed workers had high gastrointestinal cancer SMRs (a phenomenon not observed consistently in other cohorts). We agree with experts ${ }^{62}$ that the studies cannot be interpreted in terms of exposure-response. No effort at quantification would be justified.

Obviously, there is no common slope relating lung cancer SMRs to accumulated asbestos exposure. The ML point estimates of relative slope in table 3 varied from effectively zero to 6.9 , whereas one parameter slopes varied from the very small to $13 \cdot 5$. It should also be borne in mind that different periods over which the cohort was followed up and exposures were accumulated mean that comparisons are often not strictly valid (see refs 14 and 15 for the effects of such differences in a single cohort). These effects were, however, generally fairly small compared with the differences between studies. Differences may also have arisen from fibre type and dimensions, and process. Rationalisation is attempted elsewhere. ${ }^{22} 30$

\section{Conclusion}

We have shown that, although in most cohort studies of asbestos workers the relation between lung cancer SMR and accumulated asbestos exposure is effectively linear, the line often does not pass through an SMR of unity for zero exposure. The relative slope $(b / a)$, which we consider the more meaningful on similar grounds to those of Acheson and Gardner - who used our estimates-is usually quite different (sometimes steeper, sometimes more shallow) from the slope of the line forced through SMR $=1$ when $x=0$. Further, the variation in the relative slopes for different cohorts is much less than that for one parameter slopes.

We thank the German Federal Health Office (BGA) for inviting one of us (FDKL) to a symposium in Berlin in February 1982, because the proceedings reminded us of the problem and emphasised the urgency of its solution." We are grateful to Professor E D Acheson, Professor G Berry, Sir R Doll, Dr M L Gardner, and Professors Alison and C McDonald for encouragement to prepare this paper.

Requests for reprints to: Professor F D K Liddell, Department of Epidemiology and Biostatistics, McGill University, 1020 Pine Avenue West, Montreal, PQ, Canada H3A 1A2.

\section{References}

' Acheson ED, Gardner MJ. The ill effects of asbestos on health. Asbestos, Final report of the advisory committee 2. London: HMSO, 1979:7-83.

${ }^{2}$ Acheson ED, Gardner MJ. Asbestos: scientific basis for environmental control of fibres. In: Wagner JC, ed. Biological effects of mineral fibres. Lyon: International Agency for Research on Cancer, 1980:737-54. (Scientific publication No 30.)

${ }^{3}$ McDonald JC. Asbestos-related disease: an epidemiological review. In: Wagner JC, ed. Biological effects of mineral fibres. Lyon: International Agency for Research on Cancer, 1980:587-601. (Scientific publication No 30.) 
4 Nicholson WJ. Criteria document for Swedish occupational standards: asbestos and inorganic fibres. Arbete och Hälsa 1981;17:1-103.

${ }^{5}$ Schneiderman ML, Nisbet ICT, Brett SM. Assessment of risks posed by exposure to low levels of asbestos in the general environment. Bundesgesundheitsamtes-Berichte 1981;4:3/1$3 / 28$.

- Acheson ED, Gardner MJ. Asbestos: the control limit for asbestos. London: Health and Safety Commission, 1983.

${ }^{7}$ Peto J. The hygiene standard for chrysotile asbestos. Lancet 1978;i:484-9.

${ }^{8}$ Liddell FDK, McDonald JC, Thomas DC. Methods of cohort analysis: appraisal by application to asbestos mining. Journal of the Royal Statistical Society A 1977;140:469-91.

${ }^{9}$ Liddell FDK. Some new and revised risk extrapolations from epidemiological studies on asbestos workers. In: Fischer $\mathbf{M}$, Meyer $\mathrm{E}$, eds. The assessment of environmental asbestos risks. (Symposium at the Federal Health Office, Berlin-Dahlem, 17-19 February 1982, with additional material.) München: MMV Medizin Verlag, 1984: BGA-Schriften 2/84, 176-9.

${ }^{9}$ Hanley JA, Liddell FDK. Fitting relationships between exposure and SMRs. J Occup Med (in press).

${ }^{10}$ Enterline P, DeCoufle P, Henderson V. Mortality in relation to occupational exposure in the asbestos industry. $J$ Occup Med 1972;14:897-903.

" Henderson VL, Enterline PE. Asbestos exposure: factors associated with excess cancer and respiratory disease mortality. Ann NY Acad Sci 1979;330:117-26.

${ }^{12}$ Weill H, Hughes J, Waggenspack C. Influence of dose and fibre type on respiratory malignancy risk in asbestos cement manufacturing. Am Rev Respir Dis 1979;120:345-54.

${ }^{13}$ McDonald JC, Liddell FDK, Gibbs GW, Eyssen GE, McDonald AD. Dust exposure and mortality in chrysotile mining, 191075. Br J Ind Med 1980;37:11-24.

14 McDonald AD, Fry JS, Woolley AJ, McDonald JC. Dust exposure and mortality in an American factory using chrysotile, amosite, and crocidolite in mainly textile manufacture. $\mathrm{Br} J$ Ind Med 1983;40:368-74.

is McDonald AD, Fry JS, Woolley AJ, McDonald JC. Dust exposure and mortality in an American chrysotile textile plant. Br J Ind Med 1983;40:361-7.

${ }^{16}$ Dement JM, Harris RL Jr, Symonds MJ, Shy C. Estimates of dose-response for respiratory cancer among chrysotile asbestos textile workers. Ann Occup Hyg 1982;26:869-87.

${ }^{17}$ McDonald AD, Fry JS, Woolley AJ, McDonald JC. Dust exposure and mortality in an American chrysotile asbestos friction products plant. $\mathrm{Br} J$ Ind $\mathrm{Med}$ 1984;41:151-7.

${ }^{18}$ Seidman H, Selikoff IJ, Hammond EC. Short-term asbestos work exposure and long-term observation. Ann NY Acad Sci 1979;330:61-89.

${ }^{19}$ Finkelstein MM. Mortality among employees of an Ontario asbestos-cement factory. Am Rev Respir Dis 1984;129:75461.

${ }^{20}$ Berry G. The analysis of mortality by the subject-years method. Biometrics 1983;39:173-84.

${ }^{21}$ Liddell FDK. Simple exact analysis of the standardised mortality ratio. J Epidemiol Community Health 1984;38:85-8.

${ }^{22}$ McDonald JC. Mineral fibres and cancer. Ann Acad Med Singapore 1984;13 (suppl): No2, 345-51.

${ }^{23}$ Berry G. Dose-response in case-control studies. J Epidemiol Community Health 1980;34:217-22.

${ }^{24}$ Nicholson WJ, Selikoff IJ, Seidman H, Lilis R, Formby P. Longterm mortality experience of chrysotile miners and millers in Thetford Mines, Quebec. Ann NY Acad Sci 1979;330:11-21.

${ }^{25}$ Selikoff IJ, Hammond EC, Seidman H. Mortality experience of insulation workers in the United States and Canada, 19431976. Ann NY Acad Sci 1979;330:91-116.

${ }^{26}$ Newhouse ML, Berry G. Patterns of mortality in asbestos factory workers in London. Ann NY Acad Sci 1979;330:53-60.

${ }^{27}$ Peto J. Lung cancer mortality in relation to measured dust levels in an asbestos textile factory. In: Wagner JC, ed. Biological effects of mineral fibres. Lyon: International Agency for Research on Cancer, 1980:829-36. (Scientific publication No 30.)

${ }^{28}$ Berry G, Newhouse ML. Mortality of workers manufacturing friction materials using asbestos. $\mathrm{Br} \mathrm{J}$ Ind Med 1983;40:1-7.

${ }^{29}$ Albin M, Jakobsson K, Englander V, et al. Mortality and cancer morbidity in a cohort of asbestos-cement workers. In: VIth International Pneumoconiosis Conference 1983. Geneva: International Labour Office, 1984:825-9.

${ }^{30}$ Liddell FDK. Rationalisation of different slopes in the linear relationship of asbestos exposure and lung cancer SMRs. Rev Environ Health (in press). 